\title{
The Potential of Non-standard Pulsation Models for Asteroseismology
}

\author{
T. Collins ${ }^{1}$, J. Guzik ${ }^{1}$ \\ ${ }^{1}$ Los Alamos National Laboratory
}

\begin{abstract}
The process of building seismic models of observed stars that match the observed pulsation frequencies often proves to be a difficult one. The outputs of computational codes used to perform these calculations match observational data fairly well for the sun, but fail to do so as well for larger-mass stars. This suggests that the codes are neglecting potentially significant processes that occur more strongly in larger stars. We examine some hydrodynamically motivated modifications to the standard stellar models that might help to bring theoretical calculations into agreement with observational data. The analysis of Press (1981) and Press \& Rybicki (1981) is applied to the case of the $\delta$ Scuti star FG Vir, and presented.
\end{abstract}

\section{Introduction}

Current stellar evolution and pulsation models rely on a number of simplifying assumptions in order to make the problem of stellar modeling more tractable, especially when dealing with the hydrodynamics of stellar interiors. Stars exhibit a wide variety of hydrodynamic processes that affect the evolution and structure of a star to some extent, while most stellar evolution and pulsation models neglect the majority of these effects in favor of computational efficiency and simplicity. For example, the mixing-length treatment of Böhm-Vitense (1958) still seems to be the standard in general convection treatments. Non-convective instabilities are often ignored altogether in stellar evolution modeling.

This set of hydrodynamic approximations has worked quite well for the solar case. Despite the recently increased discrepancy between solar models and helioseismology with the new solar abundances these assumptions still yield far better results for the sun than they do for $\delta$ Scuti stars. Previous attempts 
at modeling the $\delta$ Scuti star FG Vir by Breger et al. (1999), Templeton et al. (2001), and Guzik et al. (2004) have met with difficulty and have yet to produce a model that can match all the frequencies observed. Templeton et al. (2001) and Guzik et al. (2004) however both had some success in improving the agreement between the predicted and observed frequencies via increased mixing between the convective core and the stable envelope. In the case of Guzik et al. (2004) core mixing was introduced into the model, not through the use of a convective overshooting parameter, as was done by Templeton et al. (2001), but instead by applying a large opacity multiplier from the core out into the radiative transport region. This multiplier caused approximately $0.8-0.9 H_{p}$ overshooting. However Deupree (2000) found in 2-D hydrodynamic simulations that for stars with under $3 M_{\odot}$ overshooting was only found to extend to $0.3 H_{p}$. Even for more massive stars Deupree (2000) found that mixing extended only to a height of $0.45 \mathrm{H}_{p}$. The amount of overshooting induced by Guzik et al. (2004) was probably too high; however the level of mixing might be justified as there are other processes that could mix hydrogen-rich envelope material into the core.

In the solar case, agreement between theoretical and observed pulsation frequencies and sound speed profiles was worsened by a downward revision of the solar metallicities. This has prompted a large number of papers examining a range of possible modifications to the solar models that could restore the previous agreement. Bahcall et al. (2005) found that a small opacity increase of roughly $10 \%$ between $2 \times 10^{6} \mathrm{~K}$ and $5 \times 10^{6} \mathrm{~K}$ (the temperature regime in the region below the convective zone) very nearly restored the agreement that had been lost in the sound speed profiles, predicted surface helium abundance, and the depth of the convective zone.

In the two separate cases of both the Sun and FG Vir opacity modifications near the boundaries of convective regions inserted into otherwise standard evolution and pulsation codes have resulted in improved agreement between predictions and observations. This suggests the possibility of a potentially significant hydrodynamic effect in the stable layers near the boundaries of the convective regions. This possibility was brought to our attention by Arnett, Meakin, and Young (private communication, in preparation). With this possibility in mind we have undertaken a large survey of possible models of FG Vir examining modifications near the surface convective zone and the core convective zone, both separately and in combination to see if this possibility could improve the agreement between frequency predictions and observation for this object. The results of that computational study are to be presented in Collins \& Guzik (in preparation). Our focus here is to consider the theoretical justification for that study. 


\section{Opacity Modification by $g$ modes Excited by Convective Over- shooting}

We consider now the work of Press (1981) and Press \& Rybicki (1981) (from here on P81 and PR81 respectively), who carefully examined exactly this possibility in detail. We begin with considering the characterization of the internal wave. P81 chose to represent the fluid flow of an internal wave in Cartesian coordinates to be

$$
\begin{aligned}
& v_{x}=-u \cos \alpha \sin \left(k_{V} z+k_{H} x+\omega t\right) \\
& v_{y}=0 \\
& v_{z}=u \sin \alpha \sin \left(k_{V} z+k_{H} x+\omega t\right)
\end{aligned}
$$

We wish to show that the results of their analysis are invariant under rotations of $\vec{k}$ with the constraint that $k^{2}=k_{V}^{2}+k_{H}^{2}$. The motivation for this being that in higher mass stars there are multiple convective regions to excite propagating g modes that would propagate both inward and outward. If the effective opacity increase predicted by PR81 is independent of the direction of wave propagation, then it should be more broadly applicable than the limited solar case considered in P81.

As in PR81 we apply the local Boussinesq approximation so that $\nabla \cdot \vec{v}=0$ which gives

$$
\tan \alpha=\frac{k_{H}}{k_{V}}
$$

so that $\alpha$ is the angle between the wave vector $\vec{k}$ and the z-axis. As described by $\mathrm{P} 81$ we can estimate $\alpha$ if we can estimate $\omega$. We can do this by using the results for $k_{V}$ and $k_{H}$ given by $\mathrm{P} 81$

$$
\begin{gathered}
k_{H} \equiv\left[\frac{l(l+1)}{r^{2}}\right]^{1 / 2} \\
k_{V} \equiv k_{H}\left[\frac{N^{2}}{\omega^{2}}-1\right]^{1 / 2}
\end{gathered}
$$

We can estimate $\omega$ from the convective turn over frequency.

We apply a coordinate transformation so that the wave vector lies along one axis.

$$
\left[\begin{array}{l}
\chi \\
\gamma \\
\zeta
\end{array}\right]=\left[\begin{array}{ccc}
\cos \alpha & 0 & -\sin \alpha \\
0 & 0 & 0 \\
\sin \alpha & 0 & \cos \alpha
\end{array}\right]\left[\begin{array}{l}
x \\
y \\
z
\end{array}\right]
$$


We transform equations (1-3) into this coordinate system where

$$
\begin{aligned}
& v_{\chi}=-u \sin (k \zeta+\omega t) \\
& v_{\gamma}=0 \\
& v_{\zeta}=0
\end{aligned}
$$

Thus for an internal wave flow the actual fluid flow is always perpendicular to the wave vector.

The effective modification to the opacity from P81 is given by

$$
<\kappa_{i j}>=\frac{\kappa_{0}}{4 \omega^{2}}\left(\sigma_{i k} \sigma_{k j}+2 \sigma_{i k} \sigma_{j k}+\sigma_{k i} \sigma_{j k}\right)
$$

where

$$
\sigma_{i j}=\frac{\partial v_{i}}{\partial x_{j}}
$$

which gives

$$
\begin{gathered}
\sigma_{i j}=\left(\begin{array}{ccc}
0 & 0 & -u k \\
0 & 0 & 0 \\
0 & 0 & 0
\end{array}\right) \\
<\kappa_{i j}>=\kappa_{0} \frac{u^{2} k^{2}}{2 \omega^{2}}\left(\begin{array}{ccc}
1 & 0 & 0 \\
0 & 0 & 0 \\
0 & 0 & 0
\end{array}\right)
\end{gathered}
$$

The heat flux is shown by PR81 to be

$$
F_{i}=-\kappa_{0} \frac{\partial T}{\partial x_{i}}-\kappa_{i j}\left(\frac{\partial T}{\partial S}\right)_{P} \frac{\partial S}{\partial x_{j}}
$$

which is equivalent to

$$
F_{i}=-\left[\kappa_{0} \delta_{i j}+\left(1-\frac{\nabla_{a d}}{\nabla}\right) \kappa_{i j}\right] \frac{\partial T}{\partial x_{j}}
$$

where $\nabla_{a d}$ and $\nabla$ have the usual meanings. Applying (14) to (16) gives

$$
\left[\begin{array}{c}
F_{\chi} \\
F_{\gamma} \\
F_{\zeta}
\end{array}\right]=\left[\begin{array}{c}
-\kappa_{0}\left(1+\left(1-\frac{\nabla_{a} d}{\nabla}\right) \frac{u^{2} k^{2}}{2 \omega^{2}}\right) \frac{\partial T}{\partial \chi} \\
-\kappa_{0} \frac{\partial T}{\partial \gamma} \\
-\kappa_{0} \frac{\partial T}{\partial \zeta}
\end{array}\right]
$$

Thus, the radiative flux is modified only along the axis of fluid flow ( $\chi$-axis), perpendicular to the direction of wave propagation ( $\zeta$-axis). If we assume that 
the temperature gradient is aligned with the vertical z-axis (an assumption inherent to one dimensional stellar evolution codes) then via the use of (7) it is easily shown that

$$
\frac{\partial T}{\partial \chi}=-\sin \alpha \frac{\partial T}{\partial z}
$$

This implies of course that the larger $k_{V}$ is relative to $k_{H}$, the weaker the modification of the radiative flux. Thus as a consequence of (6), higher frequency waves will more strongly alter the radiative opacity. The same is true of waves with higher $l$ values, as a consequence of (5).

The key conclusion that follows from (17) is that internal waves always act to reduce the radiative flux, regardless of the orientation of $\vec{k}$ relative to $\nabla T$. This implies that the effective opacity increase cannot be related to the inward luminosity carried by the energy flux of the propagating internal waves, because reversing $\vec{k}$ does not alter the effect. This conclusion is important for the asteroseismology of larger stars, because this effect should be seen in the stable interior of the star near both the core convective zone, and the envelope convective zone.

\section{Enhanced Mixing by $g$ modes Excited by Convective Overshooting}

We briefly consider in a similar manner the results of PR81 for the enhanced diffusion of a passive contaminant. In the case of heat transport above, it is the diffusion of entropy that is enhanced by the fluid motion, which results in the effective optical opacity increase. Similarly, the diffusion of particles is enhanced by fluid motion where

$$
F_{i}=-\left[\kappa_{0} \delta_{i j}+<\kappa_{i j}>\right] \frac{\partial \theta}{\partial x_{j}}
$$

where $\theta$ is the concentration of some particle species. As above it follows that

$$
\left[\begin{array}{c}
F_{\chi} \\
F_{\gamma} \\
F_{\zeta}
\end{array}\right]=\left[\begin{array}{c}
-\kappa_{0}\left(1+\frac{u^{2} k^{2}}{2 \omega^{2}}\right) \frac{\partial \theta}{\partial \chi} \\
-\kappa_{0} \frac{\partial \theta}{\partial \gamma} \\
-\kappa_{0} \frac{\partial \theta}{\partial \zeta}
\end{array}\right]
$$

where particle diffusion is enchanced along the axis of fluid motion.

In addition to the enhancement of particle diffusion, large amplitude internal waves can become unstable to subwavelength turbulence (PR81). The 
resulting mixing can extend the region of homogenous composition beyond the boundaries of the convective regions approximately out to the linear damping distance of the excited $g$ modes (P81).

\section{Conclusions and Future Work}

The most important conclusion is that stochastically excited $g$ modes that are expected to be found at the interface between convective and stable regions have the same qualitative effect of diffusion enhancement as described by PR81, regardless of the relative orientation between the wave vector and the diffusive flux being considered (represented by the angle $\alpha$ )-only the strength of the effect is dependent on alpha, not its qualitative nature.

This has important conclusions for the inclusion of this phenomenon into standard stellar models. In the case of radiation transport we expect to see enhanced diffusion of entropy which damps radiative transport. We expect to see this whether the $g$ mode causing the effect is propagating inward or outward, that is to say that the effect is independent of kinetic energy transported by the $g$ mode. Therefore it should be possible to model the effect of internal wave flow on radiative transport with a modification of optical opacities near the interfaces of all convective and stable regions. Further, we expect the region of homogenous composition around these cores to be enlarged beyond what would be expected from the overshoot heights found by Deupree (2000), especially important for the cases of larger mass stars.

In addition to the supression of radiative transport near the interfaces of convective and stable regions, we also expect to see a strengthening of particle diffusion. Keep in mind that the effect of the internal wave flow is to enhance all diffusion by some scaling factor along the direction of fluid flow. Gravitational diffusive settling should therefore be increased by this mechanism.

It would be worthwhile to produce a general theoretical formalism for expressing this effect that could be implemented in existing stellar evolution codes. Ideally such a formalism would make no assumptions about the particular convection treatment chosen and instead characterized by parameters such as the convective turnover frequency or mean flow velocity that would be predicted by the convective treatment.

\section{References}

Bahcall J.N., Basu S., Pinsonneault M., \& Serenelli A.M. 2005, ApJ, 618, 1049

Breger M., Handler G., Nather R.E., et al. 1995, A\&A, 297, 473

Breger, M., Pamyatnykh, A. A., Pikall, H., \& Garrido, R. 1999, A \&A, 341, 151

Böhm-Vitense, E. 1958, Z. Astrophys., 46, 108 
Deupree, R. G. 2000, ApJ, 543, 395 Guzik J.A., Austin B.A., Bradley P.A., \& Cox A.N. 2004, In: Kurtz D.W., Pollard

K.R. (eds.) ASP Conf. Ser. 310: IAU Colloq. 193: Variable Stars in the Local Group, 462

Press, W.H. 1981, ApJ, 245, 286

Press, W.H., \& Rybicky, G.B. 1981, ApJ, 248, 751

Templeton M., Basu S., \& Demarque P. 2001, ApJ, 563, 999 